\title{
The Research on Anti-Tumor Related Effect and Its Mechanism of Herba Sarcandrae
}

\author{
Xiuxia Wang ${ }^{1, a}$ \\ ${ }^{1}$ Luohe Medical College, Luohe, Henan, 462000 \\ ${ }^{a}$ email
}

Keywords: Herba Sarcandrae; Antitumor; Review

\begin{abstract}
Purpose of this paper is to explore Herba Sarcandrae anti-tumor effect and related mechanisms. Methods Now Chinese Journal Full-text Database (CNKI), Chinese Biomedical Literature Database (CBM), Wanfang database and VIP database aggregated from 1994 to the relevant literature and 2009, and summarized Sarcandrae anti-tumor effect and mechanism; l platelets, leukocytes, enhance the immune effect. Results Herba Sarcandrae significant anti-tumor effect and its mechanism of inducing apoptosis; Herba Sarcandrae can be increased platelets, white blood cells, enhance immunity. And chemotherapy drugs increase the discharge rate when combined with anti-tumor chemotherapy drugs, reducing its toxicity to blood and immune system. Conclusion Herba Sarcandrae inhibitory spectrum, low side effects, is widely used in clinical practice, there is a good prospect.
\end{abstract}

\section{Introduction}

Herba Sarcandrae to Chloranthaceae plant grass coral [Sarcandra glabra (Thunb1) Nakai] the whole plant. Herba Sarcandrae, alias grass coral, sarcandra, bone Chloranthus etc., only contained in the "herbal medicine prepared to be." "People's Republic of China Pharmacopoeia" 2005 edition reads: bitter, acrid, flat. The heart, liver, heat and cooling blood, blood Xiaoban, Qufeng network. For blood heat purpura, purpura, rheumatism, bruises. Modern pharmacological studies have shown that Herba Sarcandrae has analgesic, anti-inflammatory, anti-tumor, promote healing, and other activities. In this paper, a review of Chinese Journal Full-text Database (CNKI), Chinese Biomedical Literature Database (CBM), Wanfang database and VIP database from 1994 to 2009 literature on Sarcandrae anti-tumor effect and mechanism related summarized.

\section{Anti-Tumor-Associated Effects}

Anti-tumor effect of Chinese medicine, mostly tumors of consumptive disease, and the body has Archives of Jia accumulation. Body mass, Archives of Jia embolism belong to the category of blood stasis. In treatment, to expelling negatively oriented. "Lu Chuan Materia Medica" said: Herba Sarcandrae "breaking product, and pain"; "Fujian Materia Medica" words: Herba Sarcandrae "blood eliminate swelling," Modern pharmacological studies have shown Herba Sarcandrae used alone or in combination with the chemotherapy drug, liver cancer H22, sarcoma S180, nasopharyngeal tumors were significantly inhibited; and when combined with chemotherapy drugs showed significant anti-tumor is synergy.

Alone anti-tumor effects Huang Yumei and other reports, when the concentration of tetrazolium salt method (MTT method) Experiment IC50 Herba Sarcandrae injection (tumor cell growth inhibition was 50\% Herba Sarcandrae injection, respectively is $33.13,52.39 \mathrm{mg} / \mathrm{ml}$ ) can inhibit liver cancer cells Be-17402, human colon cancer cells HCT-8 cells. Herba Sarcandrae powder (2,4,8 g / kg) ig $10 \mathrm{~d}$, suppressed S180 sarcoma and Hep-A tumor inhibition rate 27.00\% 29.00\% 25.00\% $36.25 \%$ respectively; extended Ehrlich ascites carcinoma (EAC) survival time of mice, prolonging rate was $21.96 \% \sim 27.64 \%$, to $4 \mathrm{~g} / \mathrm{kg}$ obvious effect.

Synergistic antitumor effects in vitro experiments showed, 31.25,6.25,12.5 mg / ml Herba Sarcandrae injection with doxorubicin inhibition of HCT-8 cells showed additive effects; 25,50 mg / $\mathrm{ml}$ is showing synergy of high concentration Herba Sarcandrae injection with more significant role. 
Wang Jin and other used Herba Sarcandrae powder (2,4,8 g / kg), respectively, and cyclophosphamide (CTX), fluorouracil (5-Fu), adriamycin (ADM) Joint ig $10 \mathrm{~d}$, results significantly improved CTX, 5-Fu, AMD S180 mice inhibition rate, increasing the efficiency of $11.17 \%$ - 39.80\%; combined with 60Co, efficiency rate of $17.69 \%$ - 26.71\%. The mice were divided into hepatoma H22 model Herba Sarcandrae injection group $(1.5 \mathrm{~g} / \mathrm{kg}, 3.0 \mathrm{~g} \mathrm{/} \mathrm{kg}$, intraperitoneally), 5-Fu group and the combination group, continuous administration of $10 \mathrm{~d}$, the results showed that the inhibitory combination group was significantly higher than Herba Sarcandrae injection group and 5-Fu group, showed significant synergy. Liver cancer H22, S180 sarcoma mouse model, intravenous injection Sarcandrae volatile oil (SGE, 18.3,36.6 mg / kg) and cyclophosphamide (CTX) $12 \mathrm{~d}$, the inhibition rate was significantly higher than that with CTX, increase the rate of $36.83 \% \sim 51.65 \%$; oral SGE $(73.2 \mathrm{mg} / \mathrm{kg}$ ) in parallel with the CTX to H22, S180 inhibition rate also significantly increased in mice, increase the rate of $23.09 \% \sim 29.5 \%$.

To improve blood components and enhance the immune anti-tumor chemotherapy drugs on blood and immune system strong side effects, including thrombocytopenia is more serious complications. Herba Sarcandrae owned by the liver, in the classification of modern medicine belonging to the immune system, liver and can improve immunity. Modern pharmacological studies have shown that Herba Sarcandrae combined with chemotherapy drugs, radiotherapy and chemotherapy can reduce the amount of medicinal, reduce blood and immune system toxicity.

Experimental results show that improving blood components, SGE $(18.3,27.5,36.6 \mathrm{mg} / \mathrm{kg})$ in combination with CTX, CTX can cause significantly increased platelet decline; SGE (27.5,36.6 mg / kg) in combination with CTX CTX significantly antagonized white blood cell, reduce the toxicity of chemotherapy drugs. Herba Sarcandrae injection with 5-FU in combination with, can significantly correct the 5-FU-induced leukocyte and platelet decline. NORMAN, etc. with Herba Sarcandrae injection 0.2,1.0,2.0 g / kg intraperitoneal injection of $14 \mathrm{~d}$ before gastric FC mouse model, results showed significantly elevated WBC. Bell Founding reports give Babl / c mice fed Sarcandra extract (0.25 g / only .d) 12 d, while intraperitoneal injection of granulocyte colony-stimulating factor (10Ll / kg, every other day, three times), significantly improve intraperitoneal injection of 5-FU-induced thrombocytopenia, leukopenia; XU Guoliang, etc. with Herba Sarcandrae polysaccharides, coumarin, total flavonoids respectively 0.058,0.13,0.27 g / kg ig $13 \mathrm{~d}$, the results significantly Sarcandrae polysaccharide increased immune thrombocytopenia model Balb / c mice peripheral blood platelets;

Enhance the immune carbon clearance test showed Sarcandrae sheet 1.8,3.6 g / kg ig 5 d, 3.6 g / $\mathrm{kg}$ in mice can significantly improve the carbon clearance index. NORMAN reported Herba Sarcandrae injection 0.2,1.0,2.0 g / kg intraperitoneally $14 \mathrm{~d}$ before gastric FC model mice, can significantly liter mouse spleen and thymus index. Golden root found Herba Sarcandrae sheet $0.0405 \mathrm{~g} / \mathrm{kg}$ gavage, $1 / \mathrm{d}$ for 4 weeks, significantly increased liver damage in rats dimethyl nitrosamine spleen wet weight, body spleen rate; reduce immunoglobulin $G$, increase albumin and immunoglobulin A / G ratio; increased plasma total SOD (T-SOD), reduced lipid peroxidation LPO. He Rongrong reported Herba Sarcandrae extract 125,500 mg / kg \# d ig 5 d, significantly increased restraint stress model mice spleen index increased spleen lymphocytes; Flow cytometry showed that significantly increased splenic T lymphocytes cells Th / Ts, increased spleen NK cells, NKT cells and NK / NKT. XU Guoliang, etc. with Herba Sarcandrae polysaccharides, coumarin, total flavonoids were to $0.058 \mathrm{~g} / \mathrm{kg}, 0.13 \mathrm{~g} / \mathrm{kg}, 0.27 \mathrm{~g} / \mathrm{kg}$ ig $13 \mathrm{~d}$, results showed Sarcandrae polysaccharide can significantly increase the immunity thrombocytopenic purpura thymus index.

Protective effects of conventional radiation therapy combined with radiotherapy and 1.3 when the head and neck cancer patients are most commonly used and most classic radiation pattern. In the course of radiotherapy target area is exposed to the parotid gland, subjected to high doses of radiation, so there are different degrees of salivary gland dysfunction or permanent loss. Modern pharmacological experiments show Herba Sarcandrae parotid early radiation injury has a protective effect and helps repair and regenerate the gland. Male guinea pigs are divided into normal, single control group, drug control group, using the $\mathrm{C}$ ray conventional fractionated irradiation localized exposure to 1 / d, 5 times / week for $60 \mathrm{~d}$, and give medicine according to the week prior to 
irradiation from gavage group Sarcandra extract $0.212 \mathrm{mg} / \mathrm{g}$. The results showed that compared with the control group of single Herba Sarcandrae can inhibit the parotid gland atrophy and interstitial fibrosis; a significant increase in the weight of the parotid gland, inhibiting reducing parotid area; significant inhibition of inflammatory cells. Therefore, to reduce the radiation-induced inflammation may be one of the protective mechanisms. Wei Bo and other 46 patients with nasopharyngeal carcinoma were divided into Herba Sarcandrae group (25 cases) and control group (21 cases). Two groups were given the same radiotherapy; Herba Sarcandrae group $3 \mathrm{~d}$ before the start of treatment to the end, to give $20 \mathrm{~g}$ per day Sarcandrae decoction made of $100 \mathrm{ml}$. The results show that treatment can significantly reduce the swelling section acute chemotherapy toxicity, reduced radioactive dry mouth. Feng Shui Tips Herba Sarcandrae extract antioxidant injury.

\section{Anti-Tumor-Associated Mechanisms}

Treatment antitumor mechanisms to induce apoptosis of tumor cells has become a new way to treat cancer, it is also an important indicator to assess the efficacy of anticancer drugs. Recent studies have found that traditional Chinese medicine can induce apoptosis of tumor cells. Modern pharmacological studies have shown that Herba Sarcandrae can promote tumor cells to enter and arrest in G1 phase and induce apoptosis. Kangmin etc. with Herba Sarcandrae 95\% alcohol extract administered to rats fed, medicated serums, MTT experiments show Herba Sarcandrae inhibit nasopharyngeal carcinoma cell lines in vitro CNE1, CNE2, TWO3, C666-1 cells, flow cytometry (FCM) and inverted microscope show Herba Sarcandrae can induce apoptosis, cell cycle distribution, the majority of cell cycle arrest in G1 phase. Zhao Yi et al [17] reported that administration of human gastric cancer SGC-7901 tumor Balb / c athymic nude mice intravenously Herba Sarcandrae injection (1.5,3 g / kg \# d) 14 d, FCM analysis showed Herba Sarcandrae injection can promote the generation of apoptotic bodies, inducing apoptosis, increased tumor early apoptotic and late apoptotic cells. Kangmin etc. with Herba Sarcandrae 70\% ethanol extract 5 mg / (kg \# d) giving CNE1, CNE2 xenograft model mice fed ig $30 \mathrm{~d}$, significantly reduced tumor tissue Bc-l2 protein, Bax protein increased ; electron microscope Herba Sarcandrae group showed more apoptotic morphological changes; TUNEL assay showed Herba Sarcandrae apoptotic index was significantly increased; FCM showed Herba Sarcandrae group G0 / G1 phase cells increased apoptosis rate increased.

Platelet clock mechanism and other Founding give Babl / c mice fed Sarcandra extract (0.25 g / only \#d) $12 \mathrm{~d}$, while intraperitoneal injection of granulocyte colony-stimulating factor (CSF10Ll / $\mathrm{kg}$, every other day, three times ), mouse femur bone marrow biopsy show bone marrow hyperplasia, megakaryocytes, suggesting Sarcandrae prevent possibly by reducing the inhibition of chemotherapy on bone marrow system, speed up recovery of bone marrow megakaryocyte hematopoietic function peripheral platelet reduction mechanism of action, thereby preventing chemotherapy thrombocytopenia.

\section{Anti-Tumor Effects Associated Clinical Application}

Pharmacological experiments showed anti-tumor medicine Herba Sarcandrae wide range of toxicities, and can effectively enhance immunity, improve blood system, now widely used in clinical anti-tumor, improve immunity, up platelets.

Anti-tumor Huang Zhifeng, etc. to give advanced prostate cancer 26 cases twice daily intramuscular injection Herba Sarcandrae, $2 \mathrm{ml} /$ day, 15 months for a course of treatment, while giving oral flutamide, the control group was treated with flutamide tumor. Two groups were 3 months, 9 months, 15 months, measured serum prostate specific antigen (PSA), F-PSA, the results show a significant reduction Sarcandrae PSA, F-PSA / PSA and IPSS; improve the clinical symptoms improve the quality of life of patients. Advanced gastric cancer cases, 32 cases daily intravenous infusion of $20 \mathrm{ml}$ Herba Sarcandrae, once every $90 \mathrm{~d}$, and the control group using the same chemotherapy regimen. The results show Herba Sarcandrae can significantly relieve cancer pain patients; weight gain, improve quality of life; a significant reduction of bone marrow 
suppression of chemotherapy drugs, liver, kidney and gastrointestinal side effects of drugs.

Improve blood and strengthen the immune system daily intravenous injection Herba Sarcandrae $10 \mathrm{~m}, \mathrm{l}$ once every $15 \mathrm{~d}$, can significantly relieve pain in patients with cancer, which Sarcandrae restore the immune system to increase body resistance related. Gong Zhendong found that $1 / \mathrm{d}$ intravenous injection Herba Sarcandrae $20 \mathrm{~m}$, l total of $60 \mathrm{~d}$, while giving oral prednisone and prednisone alone as a control group, the results showed a significant increase Sarcandrae primary thrombocytopenic purpura thrombocytopenic patients. Li Wei has been reported daily intravenous injection Herba Sarcandrae 10 m, l once every 15 d, can be significantly increased class rheumatoid arthritis patients hemoglobin (Hgb), red blood cell count (RBC); reduced erythrocyte sedimentation rate (ESR), reducing C- reactive protein (CRP). Huang Yi Bo, reported 31 cases of nasopharyngeal carcinoma patients daily intravenous infusion of $16 \mathrm{ml}$ Herba Sarcandrae injection, once every $30 \mathrm{~d}$, while giving radiotherapy. Results Herba Sarcandrae can significantly reduce radiation-induced skin damage, nausea, vomiting, mucositis, and leukopenia. KPS increased significantly compared with the control group, the rate of rise of $84.73 \%$. Li Qing reported Herba Sarcandrae sheet can significantly improve the immune function of postoperative chemotherapy of colorectal cancer cells in patients after 35 cases of colorectal cancer patients undergoing chemotherapy, while giving Sarcandrae sheet $0.354 \mathrm{~g} 9 / \mathrm{d}$. The results show that after chemotherapy Herba Sarcandrae group CD3 +, CD4 +, CD4 / CD8 ratio, and NK significantly higher than the control group.

\section{Prospects}

By summarizing the literature found Sarcandrae antitumor effect is obvious, but its active ingredient inhibiting tumor is unclear. Studies have shown that the main component of Herba Sarcandrae are flavonoids, coumarins, organic acids, lactones, volatile oil and trace elements. But what kind of specific components or monomers anti-tumor effect requires further experimental study. Compared with chemotherapy drugs, anti-tumor effect Sarcandrae alone the poor. So often clinically the two combined to play Sarcandrae immunity in reducing the amount of chemotherapy and a pharmaceutically acceptable toxicity. Which enhances the immune mechanism, there is speculation that Herba Sarcandrae contain high zinc can improve thymus

Normal development and to promote the secretion of thymosin, adjusted so as to achieve the role of cellular immunity. But the mechanism has yet to be deeper discussion. Clinically, Sarcandrae also widely used anti-bacterial inflammation, promote healing and so on, so it has a high value, application prospects.

\section{Acknowledgements}

This is the 2015 Henan Province Science and Technology Department of Henan Province basis frontier planning project milestones. Item Number: 152300410015.

\section{References}

[1] Huang Yumei, Zhao Yi, Yang Yanping, et Herba Sarcandrae Injection Antitumor and Experimental Research and doxorubicin combination of Chinese Medicine and Pharmacology, 2007, 18 (3): 200-201.

[2] Wang Jin, Yang Feng, Shen Xiang Experimental Research Herba Sarcandrae antitumor Zhejiang Traditional Chinese Medicine, 1999,10: 450-451.

[3] Zhao Yi, Chen Qi, Sun Youzhi, and other anti-tumor effect Herba Sarcandrae United Fluorouracil of Henan Medical College newspaper, 2008,23 (2): 30-32.

[4] Lu Gui-yuan, Chen Su-hong, Zhang Yuan, etc. Sarcandrae synergy and attenuation of volatile oil on tumor-bearing mice treated by Zhejiang University of Traditional Chinese Medicine, 2009,33 (1): 116-118.

[5] NORMAN Ulrich, blue Fengying, such as the role Herba Sarcandrae injection before 
anti-mouse gastric FC and toxicity of Chinese Medicine and Pharmacology, 2003,14 (3): 169-171.

[6] Bell Founding Liutian Hao Chen Yunxian research, prevention and treatment of chemotherapy Herba Sarcandrae thrombocytopenia herbs, 2005,28 (1): 35-37.

[7] XU Guoliang, Xiaobing Hua, Chen Qi and its impact Herba Sarcandrae separate parts of immune thrombocytopenic purpura mouse platelets of Experimental Traditional Chinese, 2005, 11 (4): 33-35.

[8] Jiang Weizhe, Kong Xiaolong affect Herba Sarcandrae piece of malignancies and immune function of Guangxi Medical University, 2001,18 (1): 39-42.

[9] gold roots experimental study on the role of Herba Sarcandrae toxic liver injury in rats dimethyl nitrosamine intervention in the Shanghai Traditional Chinese Medicine, 1998, 5: 43-44.

[10] He Rongrong, Wang, Li Yifang sarcandra improve the role of traditional Chinese medicine extracts on immune function in mice stress load Traditional Chinese Medicine, 2009,34 (1): 100-102.

[11] Qin Jian, Tengjia An, Wang Rensheng Herba Sarcandrae experimental study of radiation injury Early parotid gland extract Chinese Radiological Health, 2008,17 (3): 269-271.

[12] Wei Bo, Wang Rensheng, Qin Jian, et Herba Sarcandrae feng shui extract reduce the clinical efficacy of radioactive dry mouth Guangxi Pharmaceutical University, 2009, 26 (2): 206-207.

[13] Reed JC Apoptosis targeted therapies for cancer Cancer cel, 2003, 3 (1): 171.

[14] AlisonMR, Sarraf CE. Apoptosis regulation and relevance to tox-icology. Hum ExpToxico, 1 1995,14 (3): 2341.

[15] Zhao Aiguang, Yangjin Kun, Zhao Hailei. A preliminary study the four gentlemen decoction inducing apoptosis in nude mice transplanted with human gastric cancer cells, 2001,20 (2): 1641.

[16] Kang Min, Tang Anzhou, steel beams, etc. Sarcandrae Extraction on nasopharyngeal carcinoma cell proliferation inhibition of Guangxi Medical University, 2008,25 (3): 347-348.

[17] Zhao Yi, Sun Youzhi, Chen Qi. Herba Sarcandrae injection on the growth of human gastric cancer SGC-7901 Inhibition and induction of apoptosis in tumor research. Chinese pharmacy, 2009, 20 (6): 412-414.

[18] Kangmin, Tangan Zhou Liang Gang Herba Sarcandrae nasopharyngeal carcinoma in nude mice and telomerase activity of Clinical Journal of Otorhinolaryngology Head and Neck, 2008, 22 (24): 1132-1137.

[19] Huang Zhifeng Lai sea mark, Ye had influence Herba Sarcandrae injection on 26 cases of advanced prostate cancer, PSA, F-PSA TCM research, 2006,19 (11): 25-26.

[20] Guo Yuefeng, Huang Li, Wang Shenghu Herba Sarcandrae injection combined with chemotherapy Traditional Chinese Medicine treatment of advanced gastric cancer in 32 cases, 2003,44 (6): 134.

[21] Yang Huanbiao Herba Sarcandrae injection in the treatment of cancer pain 60 cases of Chinese medicine, 2008,17 (20): 59-60.

[22] Gong Zhendong, Li Cuijuan. Herba Sarcandrae injection and prednisone idiopathic thrombocytopenic purpura Clinical Observation of 36 Cases of Shaanxi University of Chinese Medicine, 2007, 30(6): 20.

[23] Li Wei have influence Herba Sarcandrae blood injection on patients with rheumatoid arthritis and immune system Henan Traditional Chinese Medicine, 2005,25 (4): 74.

[24] Huang Yi Bo, Zhang Yurong Herba Sarcandrae injection combined with radiotherapy George nasopharyngeal Chinese clinicians, 2005,33 (6): 63. 
[25] Hao Li Ching Cheng Lijun, Zhenhong impact Herba Sarcandrae tablets on postoperative chemotherapy of colorectal cancer patient's immune function Shanghai Traditional Chinese Medicine, 2008,29 (9): 420-421.

[26] Li Yuan, Zhao mid-stream Sarcandrae chemical components and pharmacological effects and quality control studies fact sheets Chinese Pharmaceutical Affairs, 2008,22 (11): 1005. 